$(n=3)$ and RC-3095 $(0.05 \mu M-10 \mu M)(n=4)$ was assessed by Sulforhodamine $\mathrm{B}$ assay in $24 \mathrm{~h}, 48 \mathrm{~h}$ and $72 \mathrm{~h}$. Invasion assay with FLS was performed using a Matrigel-coated transwell system over $24 \mathrm{~h}$ in two different experimental arms: first, FLS were treated with GRP $(10 \mu \mathrm{M})$, RC-3095 $(1 \mu \mathrm{M})$ or GRP+RC-3095 (GRP $10 \mu \mathrm{M}$ and RC-3095 $1 \mu \mathrm{M})(\mathrm{n}=6)$ and after, FLS were treated with GRP $(10 \mu \mathrm{M})$, Ly294002 $(10 \mu \mathrm{M})$, or GRP+Ly294002 (GRP $10 \mu \mathrm{M}$ and Ly294002 10 $\mu M)(n=4)$. Akt phosphorylation was assessed by WB.

Results: GRPR protein was detected in FLS both by immunocytochemistry and WB. GRP and RC-3095 treatments did not affect FLS proliferation. Exposure to GRP increased FLS invasion $(5356 \pm 1027)$ by nearly two-fold compared with untreated cells $(2845 \pm 532)(p<0.02)$, while the treatment with RC-3095 reduced FLS invasion $(1723 \pm 271)$ compared with untreated cells. Treatment with GRP+RC-3095 $(2670 \pm 499)$ reversed the invasiveness effect of GRP $(p<0.0001)$. GRP also increased phosphorylated AKT expression in FLS by $30 \%(p<0.001)$. When Ly294002 was added with GRP (7772 \pm 1200$)$, it prevented the GRP-induced increased cell invasiveness $(11036 \pm 953.6)(p<0.001)$ (Figure 1).

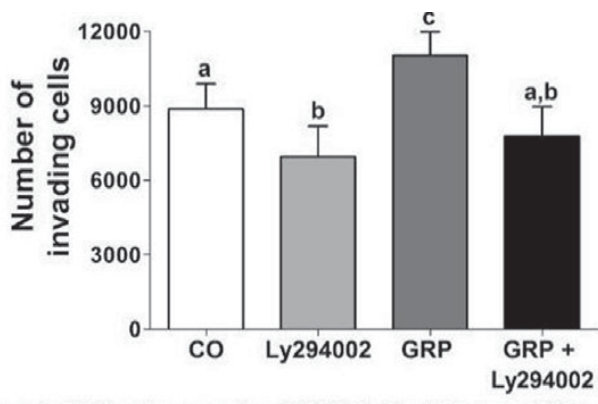

Figure 1. FLS invasion assay $(n=4)$. GRP significantly increased FLS invasion compared with untreated cells, whereas Ly294002 present opposite effect. Bars represent mean \pm SEM. Data were analyzed by generalized estimating equation (GEE) followed by Bonferroni. a) $p<0.008$ versus Ly294002; $p<0.026$ versus GRP; b) $p<0.001$ versus GRP; c) $p<0.001$ versus GRP+Ly294002

Conclusions: Our group demonstrated for the first time GRPR expression in FLS and that GRP are able to activate FLS invasion through AKT pathway. Finally, our results suggest that GRP/GRPR pathway could be relevant in the development of FLS-targeted therapy for RA.

References:

[1] Scott TL et al. Lancet 376;9746:1094-108.

[2] Bottini N, Firestein GS. Nat Rev Rheumatol 2013;9:24-33.

[3] Grimsholm O et al. Arthritis Res Ther 2005;7:R416-26.

[4] Oliveira PG et al. Arthritis Rheum 2011;63:2956-2965.

Acknowledgements: FIPE-HCPA, CAPES, CNPq.

Disclosure of Interest: None declared

DOI: 10.1136/annrheumdis-2017-eular.2484

\section{FRI0067 MICRORNA PROFILING OF MTX-TREATED FIBROBLAST-LIKE SYNOVIAL CELLS IN RHEUMATOID ARTHRITIS REVEALED A POSSIBILITY OF MICRORNA-887-3P AS NOVEL THERAPEUTIC TARGET OF RA}

N. Iwamoto, S. Fukui, T. Koga, S.-Y. Kawashiri, K. Ichinose, M. Tamai, H. Nakamura, T. Origuchu, A. Kawakami. Department of Immunology and Rheumatology, Unit of Advanced Preventive Medical Sciences, Nagasaki University Graduate School of Biomedical Sciences, Nagasaki, Nagasaki, Japan

Background: The hallmarks of rheumatoid arthritis (RA) is the expansive of fibroblast-like synovial cells (FLS) in affected joints, causing joint destruction. FLSs are resistant to programmed cell death resulting in aggressive, invasive phenotype like a cancer invades and the hyperplastic synovial tissue destroys cartilage and bone. Therefore inhibition of FLS proliferation and is one of the therapeutic targets of RA. MicroRNAs (miRNAs), a group of small non-coding RNAs, have been shown to regulate cell differentiation through regulation of gene expression post-transcriptionally. Recently, several studies reported anti-cancer drugs modulate miRNA expression and that have been considered as one of important mechanism of cellular action to drug ${ }^{1,2}$.

Objectives: To investigate the changes in microRNA expression profiles in response to MTX.

Methods: RA-FLS was treated with MTX with $1 \mathrm{nM} 48$ hours, that could inhibit IL-6 production from RA-FLS. To investigate differentially expressed miRNAs in response to MTX, we performed miRNA array analysis. Expression of miR-887-3p in response to MTX of RA-FLS was analyzed by quantitative real-time PCR. To investigate the functional role of miR-887-3p, RA-FLS was transfected with synthetic precursor miRNA (pre-miR)/inhibitors of miRNA (anti-miR) of miR-887$3 p$ using Lipofectamine and then gene expression microarray was performed. The cytokine/ chemokine production was screened by Multiplex cytokine/chemokine bead assays and confirmed by ELISA. Finally, we analysed migratory activities of RA-FLS by scratch assay.

Results: After $48 \mathrm{~h}$ of treatment with MTX, 7 miRNAs were up-regulated and 6 miRNAs were down-regulated as compared with that of untreated control. Among them quantitative real-time PCR with additional samples confirmed that miR-887-
$3 p$ was up-regulated in response to MTX treatment of RA-FLS $(1.79 \pm 0.46$-fold, $p<0.05, n=7)$. To elucidate the functional consequence of the deregulation of miR-887-3p of RA-FLS, we performed gain-and-loss of function assays with miR887-3p. Microarray analysis with gene ontology analysis revealed several genes correlated with cell signalling was modulated by miR-887-3p. Multiplex bead assay showed that overexpression of miR-887-3p decreased cytokine/chemokine production of RA-FLS such as TNF-a, GM-CSF, CCL4. Among these candidates, the secretion of GM-CSF was consistently and strongly decreased from RA-FLS transfected with pre-miR-887-3p. Furthermore overexpression of miR-887-3p reduced migratory activity of RA-FLS in scratch assay.

Conclusions: Our result showed that MTX altered micro RNA expression profiles in RA-FLS. MiR-887-3p might be downstream effector of MTX in suppression of its cytokine production and invasive phenotype. This knowledge may also be useful for the development of novel therapeutic strategies for RA based on other treatments able to boost the cellular reservoir of miR-877-3p.

References:

[1] Potenza N, Mosca N, Zappavigna S et al. MicroRNA-125a-5p Is a Downstream Effector of Sorafenib in its Antiproliferative Activity Toward Human Hepatocellular Carcinoma Cells. J Cell Physiol. 2016 Dec 16.

[2] Shah MY, Pan X, Fix LN et al. 5-Fluorouracil drug alters the microRNA expression profiles in MCF-7 breast cancer cells. J Cell Physiol. 2011; 226(7):1868-1878.

Disclosure of Interest: None declared

DOI: 10.1136/annrheumdis-2017-eular.3327

\section{FRI0068 REDUCTION OF PROLIFERATION, MIGRATION AND INVASION OF RHEUMATOID SYNOVIOCYTES BY ALL-TRANS RETINOIC ACID}

N. Mosquera ${ }^{1}$, Á. Rodríguez-Trillo ${ }^{1}$, S.B. Bravo ${ }^{2}$, A. Mera ${ }^{1}$, C. Conde ${ }^{1}$

${ }^{1}$ Experimental and Observational Rheumatology; ${ }^{2}$ Proteomic Unit, IDIS, CHUS Santiago de Compostela, Spain

Background: Fibroblast-like synoviocytes (FLS) are pivotal in inflammation and joint damage of rheumatoid arthritis (RA). These cells proliferate, become resistant to apoptosis, migrate and invade, contributing to perpetuate synovial inflammation and destruction of cartilage and bone. Current treatments of RA are focused against inflammatory factors and immune cells, however, a significant percentage of patients do not successfully respond. Combined treatments with drugs that control inflammation and with others that reverse the pathogenic phenotype of FLS could improve the prognosis of these patients. An unexplored area includes vitamin $A$ and its metabolites. These compounds modulate differentiation, development, apoptosis and proliferation. Indeed, retinoids are being successfully used in the treatment of several cancers for their anti-proliferative and pro-differentiative actions. In addition, several studies have shown a notable reduction of cell migration and invasion in different cell types after treatment with all-trans retinoic acid (ATRA). However, it is not known if ATRA could modify the migratory and invasive ability of rheumatoid synoviocytes.

Objectives: To analyse the effect of treatment with the retinoid all-trans retinoic acid in proliferation, migration and invasion of rheumatoid synoviocytes.

Methods: FLS were obtained from 8 RA patients. Cellular proliferation was determined using the CellTiter-Glo luminescent viability assay (Promega). Migration was analysed by wound healing assay, using lbidi inserts. Percentage of migrating cells was determined by Image J. Invasion was tested by the Boyden chamber method using inserts (Millicell) coated with Matrigel (BD Biosciences). Invasion was determined by quantification of Giemsa stained cells on the bottom side of the inserts under the microscope. Proteomic analysis was performed using LC-MALDI-TOF/TOF and 1-DE and 2-DE gels. MS and MS/MS data was searched against the UniProt/Swiss-Prot database of protein sequences.

Results: We analysed the effect of ATRA in the spontaneous proliferation of FLS from 8 RA patients. ATRA treatment significantly reduced proliferation at 48,72 and $96 \mathrm{~h}$ ( $p=0,005 ; p=0,002 ; p=0,04 ;$ respectively). Next, we analysed the effect of ATRA on RA FLS migration and invasion. Migration of RA FLS treated with ATRA for $96 \mathrm{~h}$ was reduced by $46 \%$ when compared with cells treated with vehicle control $(p=0,002)$. In addition, RA FLS invasion was also impaired after ATRA treatment, as the invaded area was $31 \%$ lower than in controls $(p=0,018)$. To elucidate the mechanisms underlying the effects of ATRA on RA FLS a proteomic analysis was performed. We compared the proteome of FLS from 5 RA patients treated with ATRA or with vehicle using LC-MALDI analysis. The differentially identified proteins in ATRA-treated FLS vs control FLS were Septin, Rho GTPase activating protein (ARHGAP1) and actin related protein 2 (ARP2). Validation experiments using 1-DE and 2-DE gels were performed.

Conclusions: Overall these results reveal that retinoid treatment reduces the proliferative, migratory and invasive capacity of RA FLS, indicating that this treatment could decrease joint damage and loss of function in RA patients.

Acknowledgements: Supported by grant PI14/01153 of the ISCIII (Spain), partially financed by FEDER.

Disclosure of Interest: None declared

DOI: 10.1136/annrheumdis-2017-eular.3139 